\title{
Preliminary meta-analysis of the effect of fish oil on body weight and body mass index in overweight and obese subjects does not support a link
}

\author{
C. J. Harden, M. E. Barker, J. M. Russell and B. M. Corfe \\ The University of Sheffield, Faculty of Medicine, Dentistry and Health, Beech Hill Road, Sheffield, S10 $2 R X$, UK
}

There is evidence to suggest that longer-chain polyunsaturated fatty acids (LCPUFA) may have greater appetitive effects than shorterchain, mono- and saturated fatty acids. The purpose of the following work was to undertake a meta-analysis to examine the strength of evidence for an effect of $n-3$ fish oil on body weight and body mass index (BMI).

A search of the literature was conducted using Web of Science, PubMed, Medline and Google Scholar for studies having the keywords 'fish', 'fish oil', 'oily fish', 'omega three', 'omega-3', ' $n$-3', 'body weight', 'body composition', 'BMI', 'weight reduction' or 'weight loss'. Extensive cross referencing searches were also performed and published abstracts were evaluated for inclusion. The initial search revealed fifteen studies. Studies were excluded if they were not randomised controlled trials, had not compared the effect of fish oil supplementation with another (non- $n-3)$ oil control, had not used overweight or obese subjects and had not taken pre- and postintervention measurements of body weight and BMI; six studies were rejected. Requests for data were only accepted from four research groups. Mean values of pre- and post-intervention body weight $(\mathrm{kg})$ and BMI $\left(\mathrm{kg} / \mathrm{m}^{2}\right)$ were calculated along with the standard deviation and standard error of the mean values. $\Delta$ change from baseline and the $\Delta$ difference between body weight and BMI changes were also calculated.

None of the studies reported a statistically significant effect of fish oil treatment relative to placebo. Further analysis showed no relationship between body weight change with intervention duration, or $n$-3 dosage, nor any funnel effect with study sample size.

\begin{tabular}{|c|c|c|c|c|c|c|c|c|c|}
\hline \multirow[b]{2}{*}{ Lead Author Treatment } & \multicolumn{4}{|c|}{$\Delta$ body weight $(\mathrm{kg})$} & \multicolumn{4}{|c|}{$\Delta \mathrm{BMI}\left(\mathrm{kg} / \mathrm{m}^{2}\right)$} & \multirow{2}{*}{$\begin{array}{c}\text { Reported } \\
p=\end{array}$} \\
\hline & Mean & & SD & SEM & Mean & & SD & SEM & \\
\hline \multicolumn{10}{|l|}{ Krebs $^{1}$} \\
\hline Diet + FO & -10.00 & \pm & 5.20 & 0.88 & -3.80 & \pm & 2.10 & 0.35 & \multirow[t]{2}{*}{$>0.05$} \\
\hline Diet + PO & -11.20 & \pm & 5.10 & 0.90 & -4.20 & \pm & 1.90 & 0.34 & \\
\hline \multicolumn{10}{|l|}{ Hill $^{2}$} \\
\hline FO & 0.03 & \pm & 2.14 & 0.52 & 0.00 & \pm & 0.75 & 0.18 & \multirow[t]{2}{*}{$>0.05$} \\
\hline $\mathrm{PO}$ & 0.23 & \pm & 1.20 & 0.28 & 0.08 & \pm & 0.45 & 0.11 & \\
\hline Exercise + FO & -0.20 & \pm & 2.56 & 0.64 & -0.59 & \pm & 0.91 & 0.23 & \multirow{2}{*}{$>0.05$} \\
\hline Exercise $+\mathrm{PO}$ & -1.73 & \pm & 2.63 & 0.70 & -0.07 & \pm & 0.82 & 0.22 & \\
\hline \multicolumn{10}{|l|}{$\mathrm{Kabir}^{3}$} \\
\hline Diet + FO & -1.54 & \pm & 2.44 & 0.68 & -0.22 & \pm & 1.08 & 0.30 & \multirow[t]{2}{*}{$>0.05$} \\
\hline Diet + PO & -0.64 & \pm & 2.37 & 0.63 & -0.02 & \pm & 0.53 & 0.14 & \\
\hline \multicolumn{10}{|l|}{$\mathrm{Kratz}^{4}$} \\
\hline Diet + FO & -3.07 & \pm & 4.06 & 1.13 & -1.10 & \pm & 1.44 & 0.39 & \multirow[t]{2}{*}{0.541} \\
\hline Diet + PO & -4.00 & \pm & 4.19 & 1.16 & -1.39 & \pm & 1.42 & 0.40 & \\
\hline $\mathrm{FO}=$ fish oil, $\mathrm{PO}=$ pla & & & & & & & & & \\
\hline
\end{tabular}

This analysis does not support the hypothesis that daily $n-3$ oil supplementation reduces body weight and BMI in the overweight and obese.

1. Krebs JD, Browning LM, McLean NK et al. (2006) Int J Obes 30, 1535-1544.

2. Hill AM, Buckley JD, Murphy KJ et al. (2007) Am J Clin Nutr 85, 1267-1274.

3. Kabir M, Skurnik G, Naour N et al. (2007) Am J Clin Nutr 86, 1670-1679.

4. Kratz M, Callahan HS, Yang PY et al. (2009) Nutr Metab 6, 24-8. 